$66 / 2016$

\title{
ESTUDIO COGNITIVO DE LA POLISEMIA DE 手 SHǑU MANO \\ EN CHINO Y ESPAÑOL
}

\author{
Xin Wei 魏欣 \\ Universidad de Granada \\ weixin_sofia en $126 \mathrm{com}$
}

Resumen

En el estudio de la polisemia en chino y español, el enfoque cognitivo nos indica qué tienen en común los patrones polisémicos de ambas lenguas, chino y español, de manera que se puedan hacer generalizaciones aplicables a otras lenguas y a otros fenómenos semánticos del lenguaje. Partimos de la hipótesis de que existen mecanismos universales de extensión semántica que pueden ser puestos de relieve y que pueden servir en la enseñanza y aprendizaje de lenguas.

Palabras clave: polisemia, lingüística cognitiva, comparación en chino y en español, asociación semántica.

Wei, Xin 魏欣. 2016.

Estudio cognitivo de la polisemia de '手 shǒu mano en chino y español.

Círculo de Lingüística Aplicada a la Comunicación 66, 382-401.

http://www.ucm.es/info/circulo/no66/wei.pdf

http://revistas.ucm.es/index.php/CLAC

http://dx.doi.org/10.5209/CLAC.52778

(C) 2016 Xin Wei 魏欣

Círculo de Lingüística Aplicada a la Comunicación (clac)

Universidad Complutense de Madrid. ISSN 1576-4737. http://www.ucm.es/info/circulo 


\section{Abstract}

When studying polysemy in Chinese and Spanish, a cognitive approach shows what is common in the polysemous patterns of both languages, so that we can make generalizations applicable to other languages and other semantic phenomena of language. The paper's hypothesis is that there are universal mechanisms of semantic extension that can be highlighted and can be used in teaching and learning languages.

Key words: polysemy, cognitive linguistic, compared Chinese and Spanish, semantic association.

Índice

1. Introducción 384

2. Estudio cognitivo de shǒu手 384

3. Estudio cognitivo de 'mano' 393

4. Comparación cognitiva de shǒu手 mano en chino y en español 396

4.1. Comparación de la categoría gramatical 396

4.2. Comparación de las asociaciones semánticas 397

4.2.1. Usos comunes de las asociaciones semánticas en chino y en español 397

4.2.2. Diferencias entre las asociaciones semánticas en chino y en español 399

5. Conclusión 400

Bibliografía 401 


\section{Introducción}

Este trabajo está dedicado a la comparación cognitiva sobre el término polisémico 'mano (shǒu f)' en chino y en español (sobre la polisemia en inglés para estudiantes chinos, véase Zhāng 张绍全 2010).

Sabemos que los estudios semánticos sobre la polisemia siempre han sido un tema importante para las investigaciones lingüísticas desde hace siglos. Existen muchas formas de definir la polisemia y sus clasificaciones. Aquí entendemos la polisemia como aquellas palabras que pueden tener dos o más sentidos, pero que se encuentran interrelacionados (Pottier Navarro, 1991:21).

En los estudios de lingüística cognitiva, el interés está en describir la polisemia desde nuevos puntos de vista. De acuerdo con esta disciplina, la polisemia es más que una manifestación semántica, que contiene diferentes grados de representatividad, dichas representaciones no se encuentran aisladas sino que, al contrario, están interrelacionadas por algún elemento en común (Cuenca, 2013:126).

\section{Estudio cognitivo de shǒu手}

La palabra shǒu手, por tratarse de una parte importante del cuerpo humano, tiene muchas acepciones tanto en chino como en español. Vamos a ver primero las asociaciones semánticas de este término en chino:

a. Sentido literal, en adelante, SL: mano como una parte del cuerpo humano;

b. Sentido figurado, en adelante, SF: las partes del cuerpo humano relacionadas con la mano;

c. SF: se asocia con los objetos relacionados con la mano;

d. SF: se asocia con las acciones que se pueden realizar con las manos: tocar; fabricar o producir; escribir; tomar, llevar, coger y sujetar;

e. SF: se asocia con las habilidades y los métodos, con las personas que tienen estas habilidades en sentido positivo (profesión), neutral y negativo;

f. SF: se asocia con fuerzas y poder; 
g. SF: se asocia con las relaciones familiares o cercanas;

h. SF: se usa como clasificador para contar la habilidad, la cantidad, derecho de propiedad;

i. SF: se asocia con otras fraseologías.

\begin{tabular}{|c|c|}
\hline Acepciones & Ejemplos \\
\hline $\begin{array}{l}\text { a. SL: mano como una parte del } \\
\text { cuerpo humano }\end{array}$ & $\begin{array}{l}\text { Tā de shǒu hěn piào liang (她的手很漂亮 } \\
\text { Sus manos son muy bonitas) }\end{array}$ \\
\hline $\begin{array}{l}\text { b. SF: las partes del cuerpo } \\
\text { humano relacionadas con la mano }\end{array}$ & $\begin{array}{l}\text { Shǒu zhǐ (手指dedo) } \\
\text { Shǒu wàn (手腕muñeca) } \\
\text { Shǒu zhăng (手掌palma) } \\
\text { Shǒu bì (手臂brazo) }\end{array}$ \\
\hline $\begin{array}{l}\text { c. SF: se asocia con los objetos } \\
\text { relacionados con la mano; }\end{array}$ & $\begin{array}{l}\text { Shǒu jī(手机lit. máquina de mano; fig. móvil) } \\
\text { Shǒu biăo(手表lit. reloj de mano; fig. reloj de muñeca o de pulsera) } \\
\text { Shǒu zhuó(手镯it. pulsera de mano; fig. pulsera) } \\
\text { Shǒu tí xiāng(手提箱it. maleta de mano---maletín) } \\
\text { Shǒu tí diàn năo(手提电脑lit. ordenador de mano; fig. portátil) }\end{array}$ \\
\hline $\begin{array}{l}\text { d. SF: se asocia con las acciones } \\
\text { que se pueden realizar con las } \\
\text { manos }\end{array}$ & \\
\hline $\begin{array}{l}\text { Se asocia con la acción de } \\
\text { tocar }\end{array}$ & $\begin{array}{l}\text { Shǒu yín(手畄it. mano obscena; fig. masturbación) } \\
\text { Shǒu găn(手感it. sensación de mano; fig. tacto) }\end{array}$ \\
\hline $\begin{array}{l}\text { Se asocia con la acción de } \\
\text { fabricar y producir }\end{array}$ & $\begin{array}{l}\text { Shǒu gōng(手卫lit. trabajo de mano; fig. obra manual) } \\
\text { Shǒu yì(手艺lit. técnica de mano; fig. hechura o arte) } \\
\text { Qīn shǒu(亲手lit. besar la mano; fig. hacer algo por uno mismo) } \\
\text { Shǒu yǔ(手语lit. lenguaje de mano; fig. lenguaje de signos o de } \\
\text { señas) }\end{array}$ \\
\hline $\begin{array}{l}\text { Se asocia con la acción de } \\
\text { escribir }\end{array}$ & $\begin{array}{l}\text { Shǒu chāo(手抄it. copia de mano; fig. manuscrito) } \\
\text { Shǒu găo(手稿lit. escrito de mano; fig. manuscrito) } \\
\text { Shǒu yù(手渝lit. orden de mano; fig. directiva manuscrita) }\end{array}$ \\
\hline $\begin{array}{l}\text { Se asocia con la acción de } \\
\text { tomar, llevar, coger y sujetar }\end{array}$ & $\begin{array}{l}\text { Wò shǒu(握手estrechar la mano) } \\
\text { Shǒu tí(手提llevar o tomar algo en la mano) }\end{array}$ \\
\hline $\begin{array}{l}\text { Se asocia con la acción de } \\
\text { meter }\end{array}$ & Chā shǒu(插手lit. meter la mano; fig. intervenir) \\
\hline
\end{tabular}




\begin{tabular}{|c|c|}
\hline $\begin{array}{l}\text { e. SF: se asocia con las } \\
\text { habilidades y los métodos }\end{array}$ & \\
\hline $\begin{array}{l}\text { Se asocia con las habilidades } \\
\text { y los métodos }\end{array}$ & $\begin{array}{l}\text { Miào shǒu huí chūn(妙手回春 } \\
\text { lit. con la mano hábil volver la primavera; } \\
\text { fig. (un médico experto) devolver la vida de un paciente que está al } \\
\text { borde de la muerte) } \\
\text { Shǒu dào bìng chú(手到病除 } \\
\text { lit. desaparecer la enfermedad con un toque de mano; } \\
\text { fig. un médico de alto nivel) } \\
\text { Xīn líng shǒu qiăo(心灵手巧 } \\
\text { lit. alma sensible y mano hábil; fig. (uno) inteligente y hábil) } \\
\text { Yăn gāo shǒu dī (眼高手低 } \\
\text { lit. ojos altos y manos bajas; } \\
\text { fig. tener gran ambición, pero poca capacidad para conseguirla) } \\
\text { Xīn hěn shǒu là (心狠手辣 } \\
\text { lit. corazón cruel y mano maliciosa; } \\
\text { figg. ser extremadamente cruel y despiadado) }\end{array}$ \\
\hline \multirow[t]{3}{*}{$\begin{array}{l}\text { Se asocia con las personas } \\
\text { que tienen estas habilidades } \\
\text { en sentido positivo } \\
\text { (profesión), neutral y } \\
\text { negativo; }\end{array}$} & $\begin{array}{l}\text { En sentido positivo (profesión) } \\
\text { Gē shǒu (歌手lit. mano para cantar; fig. cantante) } \\
\text { Sài chē shǒu (赛车手 } \\
\text { lit. mano para carreras de automóviles; } \\
\text { fig. piloto de Fórmula 1) }\end{array}$ \\
\hline & $\begin{array}{l}\text { En sentido neutral } \\
\text { Xuăn shǒu(选手lit. mano para elegir; fig. candidato) } \\
\text { Duō miàn shǒu (多面手 } \\
\text { lit. mano polifacética; fig. maestro polifacético) } \\
\text { Bāng shǒu(帮手lit. mano para ayudar; fig. ayudante) }\end{array}$ \\
\hline & $\begin{array}{l}\text { En sentido negativo } \\
\text { Pá shǒu (扒手lit. mano para escarbar; fig. ladrón o ratero) } \\
\text { Guì zi shǒu (会子手lit. mano para cortar; fig. verdugo) } \\
\text { Dǎ shǒu (打手lit. mano para golpear; fig. matón o verdugo) }\end{array}$ \\
\hline f. SF: se asocia con fuerza y poder & $\begin{array}{l}\text { Ȳ̄ shǒu zhē tiān (一手遮天 } \\
\text { lit. Una mano que cubre el cielo; } \\
\text { fig. ocultar la verdad al público o engañar al público mediante el } \\
\text { poder) }\end{array}$ \\
\hline
\end{tabular}




\begin{tabular}{|c|c|}
\hline & $\begin{array}{l}\text { Shǒu wú fù jī zhī lì (手无缚鸡之力 } \\
\text { lit. las manos son tan débiles que no tienen fuerza para agarrar la } \\
\text { gallina; } \\
\text { fig. ser muy débil físicamente) } \\
\text { Shǒu wú cùn tiě (手无寸铁 } \\
\text { lit. no tener ningún arma de fuego o cortopunzante en las manos; } \\
\text { fig. estar totalmente desarmado) } \\
\text { Shǒu qì (手气it. el aire de la mano; fig. la suerte al jugar a las } \\
\text { cartas) }\end{array}$ \\
\hline $\begin{array}{l}\text { g. SF: se asocia con las relaciones } \\
\text { familiares o cercanas }\end{array}$ & $\begin{array}{l}\text { Qíng tóng shǒu zú }{ }^{1} \text { (情司手足 } \\
\text { lit. tener el mismo sentimiento que hay entre las manos y los pies; } \\
\text { fig. tener una relación tan cercana entre los amigos como la que se } \\
\text { tiene con los familiares) } \\
\text { Xiōng dì rú shǒu zú (兄弟如手足 } \\
\text { lit. los hermanos son como las manos y los pies; } \\
\text { fig. tener una amistad tan cercana como el amor fraternal) } \\
\text { Shǒu xīn shǒu bèi dōu shì ròu (手心乎背都是肉 } \\
\text { lit. tanto la palma como el dorsal de la mano son carne de uno; } \\
\text { fig. no puede elegir cuál es su favorita entre las dos personas a las } \\
\text { que quiere) }\end{array}$ \\
\hline & \\
\hline
\end{tabular}

${ }^{1}$ Las expresiones parecidas son: shǒu zú zhī qíng 手足之情lit. el sentimiento de las manos y los pies; fig. el amor fraternal); shǒu zú qíng shēn 手足情罙lit. el sentimiento profundo de las manos y los pies; fig. el amor profundo y fraternal); qīn rú shóu zú 榇呼足lit. tener el mismo sentimiento como el que hay entre las manos y los pies; fig. tener una relación tan cercana entre dos amigos como las que se tienen con los familiares).

${ }^{2}$ Una expresión como esta es: xiōng dì rú shǒu zú, nü rén rú yĩ fú 兄弟如手足 女人如不服lit. el amigo es como el hermano y la esposa es como la ropa) viene de las palabras de Liú bèi 刘條año 161-223, poderoso jefe militar y emperador de Shǔ蜀en la era de los Tres Reinos de China) de la obra clásica Sān guó yăn yì三国演义(Romance de los Tres Reinos), más tarde la gente la adaptó de la siguiente forma: xiōng

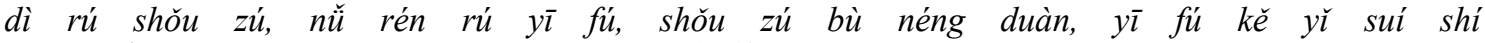
huàn 兄弟如手足，女人如不服 手足不能断，衣服可以随付换(lit. el amigo es como el hermano y la esposa es como la ropa, las relaciones entre los hermanos no se pueden romper igual que no se pueden cortar los enlaces entre las manos y las piernas, mientras tanto, las ropas pueden cambiarse en cualquier momento). 


\begin{tabular}{|c|c|}
\hline cantidad, derecho de propiedad; & \\
\hline $\begin{array}{l}\text { Clasificador para contar la } \\
\text { cantidad }\end{array}$ & $\begin{array}{l}\text { Ȳì shǒu gǔ piào (一手股票it. una mano de acciones; fig. las } \\
\text { acciones) } \\
\text { Ȳì shǒu hào pái (一手好牌una mano de cartas buenas) }\end{array}$ \\
\hline $\begin{array}{l}\text { Clasificador para contar la } \\
\text { habilidad }\end{array}$ & $\begin{array}{l}\text { Liú yī shǒu (留一手 } \\
\text { lit. quedar una mano; } \\
\text { fig. (el maestro) Al enseñar al discípulo un oficio o una habilidad, } \\
\text { guardar un truco o dos en vez de dárselo todo) } \\
\text { Lòu ȳ̄ shǒu (露一手 } \\
\text { lit. mostrar una mano; } \\
\text { fig. hacer alarde de su habilidad; lucirse en algún campo) } \\
\text { Xiě dé yī shǒu hào zì } \\
\text { (写得一手好字 } \\
\text { lit. escribir una mano caligrafías; } \\
\text { fig. (uno) tener buena o bonita letra) }\end{array}$ \\
\hline $\begin{array}{l}\text { Clasificador para contar el } \\
\text { derecho de propiedad }\end{array}$ & $\begin{array}{l}\text { Ȳ̄ shǒu zī liào (一手资料 } \\
\text { lit. materiales o recursos de primera mano; } \\
\text { fig. los últimos materiales o recursos) } \\
\text { Èr shǒu huò(二手货productos de segunda mano) }\end{array}$ \\
\hline $\begin{array}{l}\text { i. SF: se asocia con otras } \\
\text { fraseologías. }\end{array}$ & $\begin{array}{l}\text { Shǒu wǔ zú dăo (手舞足蹈 } \\
\text { lit. bailar las manos y los pies; fig. estar muy alegre) } \\
\text { Shǒu xià bài jiàng(手下败将 } \\
\text { lit. un comandante derrotado en mis manos; fig. un rival derrotado) } \\
\text { Shǒu xià liú qíng(手下留情 } \\
\text { lit. solicitar piedad a alguien con las manos; } \\
\text { fig. mostrar misericordia hacia alguien) } \\
\text { Shǒu jí yăn kuài(手知眼决 } \\
\text { lit. manos hábiles y ojos rápidos; fig. moverse o actuar con mucha } \\
\text { rapidez) } \\
\text { Máo shǒu máo jiăo(毛手毛脚 } \\
\text { lit. mano y pie ásperos; fig. descuido al sostener las cosas) } \\
\text { Zhǐ shǒu huà jiǎo(指手画脚 } \\
\text { lit. señalar la mano y dibujar el pie; } \\
\text { fig. criticar o dar órdenes de manera sumaria) } \\
\text { Gāo tái guì shǒu(高抬贵手 }\end{array}$ \\
\hline
\end{tabular}




\begin{tabular}{|l|l|}
\hline & $\begin{array}{l}\text { lit. (uno) levantar su mano privilegiada; } \\
\text { fig. (pedir a uno que) muestre su misericordia hacia otro) } \\
\text { Bái shǒu qí jiā(白手起家 } \\
\text { lit. levantar la casa con manos vacías; } \\
\text { fig. empezar a hacer algo desde cero) } \\
\text { Fān shǒu wéi yún fù shǒu wéi yŭ(翻手为云覆手为雨 } \\
\text { lit. producir nubes con un giro de la mano y la lluvia con otra; } \\
\text { fig. (uno) actuar de manera contradictoria consigo mismo) }\end{array}$ \\
\hline
\end{tabular}

Las manos (shǒu手) forman parte de las extremidades del cuerpo humano. Se encuentran en los extremos de los antebrazos y tienen cinco dedos. Están conectadas con los brazos a través de la muñeca. Por lo tanto, muchas partes que están vinculadas con la mano se nombran con este término: shǒu zh亡̆手指(dedo), shǒu wàn 手腕(muñeca), shŏu zhăng 手掌(palma).

Las manos son prensiles y el principal órgano para la manipulación física del medio, por eso, muchos objetos y sujetos también han sido nombrados con el término shǒu手: shǒujī 手机(lit. máquina de mano; fig. móvil), shǒu biăo手表(lit. reloj de mano; fig. reloj de muñeca o de pulsera), shǒu zhuó 手锣(lit. pulsera de mano; fig. pulsera), shǒu tí xiāng 手提箱(lit. maleta de mano---maletín), shǒu tí diàn năo 手提电脑(lit. ordenador de mano; fig. portátil).

La punta de los dedos contiene algunas de las zonas con más terminaciones nerviosas del cuerpo humano, son la principal fuente de información táctil sobre el entorno. Las manos pueden ser la parte más flexible para hacer movimientos y realizar muchas acciones. De esta manera, pueden asociarse con las acciones que pueden realizarse a través de ellas: tocar: shǒu găn 手感(lit. sensación de mano; fig. tacto), shǒu yín 手滛(lit. mano obscena; fig. masturbación); fabricar o producir: shǒu gōng 手工(lit. trabajo de mano; fig. obra manual), qīn shǒu亲手(lit. besar la mano; fig. hacer algo por uno

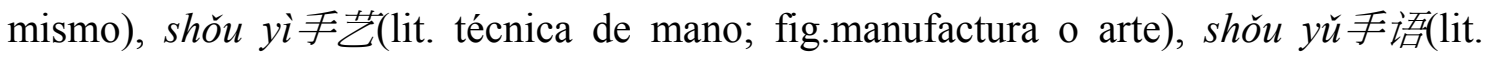
lenguaje de mano; fig. lenguaje de signos o de señas); escribir: shǒu chāo f手抄(lit. copia de mano; fig. manuscrito), shǒu găo 手稿(lit. escrito de mano; fig. manuscrito), shǒu 
$y \grave{u}$ 手渝(lit. orden de mano; fig. directiva manuscrita), etc.; tomar, llevar, coger y sujetar: wò shǒu握手(estrechar la mano), shǒu tí 手提(llevar o tomar algo en la mano).

En el budismo, existe un tipo de Buda que se llama qiān shǒu guān yīn 千手观音(Buda de mil manos). Este dios cuenta con muchas leyendas folklóricas sobre su origen; una de las más famosas es la que relata que el emperador de un antiguo reino de China tenía tres hijas, y la menor era muy creyente en el budismo, hasta que incluso se mete en un templo de clausura para estudiarlo. El padre se enfadó mucho y mandó a su gente a quemarlo, matando a los quinientos monjes que vivían dentro para obligar a su hija a volver al palacio. Poco después, el emperador cayó enfermo y le salieron 500 llagas en todo el cuerpo. Ningún médico pudo curarlo. Un mago le dijo que tendría que comer una mano y un ojo de una de sus hijas. Sin embargo, ninguna de las dos mayores querían hacer este sacrificio por su padre. La menor, como era budista creyente, quería hacerlo por el emperador con el fin de corresponder a las bondades y el amor de su padre durante años. Finalmente, el padre se recuperó. Al enterarse de todo esto el Buda Gautama, considerado fundador del Budismo, descendió del cielo y convirtió a la menor en su nueva discípula Qiān shǒu guān yīn千手观音 (Buda de mil manos), por su obediencia y amor a sus padres. Le dio mil manos con un ojo situado en el centro de cada una de ellas. Con tantas manos y ojos tendría el poder suficiente para cuidar y ayudar a los pobres del mundo profano.

A través de la leyenda mencionada, no es difícil saber que Qiān shǒu guān yīn 千手观音 (Buda de mil manos) es una figura que puede cuidar a las personas miserables del mundo profano con la ayuda de sus numerosas manos. La gente tiene tanto respeto y amor hacia esta figura, que hasta inventa un baile con el mismo nombre, que tiene mucha popularidad y éxito (véase la versión más conocida en https://www.youtube.com/watch?v=kKpljISWP2w).

Tanto por los conocimientos comúnes sobre el mundo, como por las leyendas tradicionales, sabemos que las manos pueden realizar múltiples acciones, eso quiere decir que están relacionadas con algunas habilidades. Por eso, se pueden asociar con las habilidades y los métodos: miào shǒu huí chūn妙手回春 (lit. con la mano hábil volver la primavera; fig. (un médico experto) devolver la vida de un paciente que está al borde 
de la muerte), xīn líng shǒu qiăo心灵手巧(lit. alma sensible y mano hábil; fig. (uno) inteligente y hábil), shǒu dào bìng chú 手到病除(lit. desaparecer la enfermedad con un toque de mano; fig. un médico de alto nivel), yăn gāo shǒu dī眼高手低(lit. ojos altos y manos bajas; fig. tener gran ambición pero poca capacidad para conseguirla), xīn hěn shóu là 心狠手辣(lit. corazón cruel y mano maliciosa; fig. ser extremadamente cruel y despiadado); con las personas que tienen estas habilidades en sentido positivo (profesión): gēe shǒu歌手(lit. mano para cantar; fig. cantante), sài chē shǒu赛车手(lit. mano para carreras de automóviles; fig. piloto de Fórmula I); neutral: xuăn shǒu选手 (lit. mano para elegir; fig. candidato), duō miàn shǒu多面手(lit. mano polifacética; fig. maestro polifacético), bāng shǒu帮手(lit. mano para ayudar; fig. ayudante); y negativo: pá shǒu扒手(lit. mano para escarbar; fig. ladrón o ratero), dă shǒu打手(lit. mano para golpear; fig. matón o verdugo), gui zi shǒu会/子手(lit. mano para cortar; fig. verdugo), etc.

Las acciones realizadas mediante las manos requieren el uso de la fuerza, por lo tanto, pueden asociarse con la fuerza y el poder: $y \bar{\imath}$ shǒu zhe tiān一手遮天(lit. Una mano que cubre el cielo; fig. ocultar la verdad al público o engañar al público mediante el poder), shǒu wú fù jī zhī lì 手无缚鸡之力(lit. las manos son tan débiles que no tienen fuerza para agarrar la gallina; fig. ser muy débil físicamente), shǒu wú cùn tiě手无寸铁(lit. no tener ningún arma de fuego o cortopunzante en las manos; fig. estar totalmente desarmado), shǒu qi 手气(lit. el aire de la mano; fig. la suerte al jugar a las cartas).

Las manos están conectadas por los tendones y los huesos, lo cual simboliza una relación muy cercana igual que la relación sanguínea, por eso se puede asociar con las relaciones familiares y cercanas como la amistad: qíng tóng shǒu zú 情同手足 (véase nota 1; lit. tener el mismo sentimiento que el que hay entre las manos y los pies; fig. tener una relación tan cercana entre los amigos como la que se tiene con los familiares), xiōng dì rú shǒu zú 兄弟如手足 (véase nota 2; lit. los hermanos son como las manos y los pies; fig. tener una amistad tan cercana como el amor fraternal), shǒu xīn shǒu bèi dōu shì ròu手心手背都是肉(lit. tanto la palma como el dorsal de la mano son carne de uno; fig. no puede elegir cuál es su favorita entre las dos personas a las que quiere), etc. 
Sabemos que las manos pueden contarse y también pueden contar las cosas, así este término sirve como clasificador para contar las habilidades: liú ȳi shǒu留一手(lit. quedar una mano; fig. (el maestro) Al enseñar al discípulo un oficio o una habilidad, guardar un truco o dos en vez de dárselo todo), lòu ȳ shǒu露一手(lit. mostrar una mano; fig. hacer alarde de su habilidad; lucirse en algún campo), xiě dé ȳ̄ shǒu hào $z i$ 写得一手好字(lit. escribir una mano caligrafías; fig. (uno) tener buena o bonita letra); para contar las cantidades: $y \bar{\imath}$ shǒu gǔ piào一手股票(lit. una mano de acciones; fig. las acciones), yī shǒu hào pái一手好牌 (una mano de cartas buenas); y también para contar el derecho de propiedad: $y \bar{\imath}$ shǒu zi liào一手资料 (lit. materiales o recursos de primera mano; fig. los últimos materiales o recursos), Èr shǒu huò二手货(productos de segunda mano).

Por último, también existen otras fraseologías relacionadas con este término, pero no puede conseguirse una clasificación semántica cognitiva con claridad: shǒu wǔ zú dăo 手舞足蹈(lit. bailar las manos y los pies; fig. estar muy alegre), shǒu xià bài jiàng手下败哹(lit. un comandante derrotado en mis manos; fig. un rival derrotado), bái shǒu q̌̌ jiā 白手起家 (lit. levantar la casa con manos vacías; fig. empezar a hacer algo desde cero), shǒu xià liú qíng 手下留情(lit. quedar piedad en las manos hacia alguien; fig. mostrar misericordia hacia alguien), shǒu jí yăn kuài 手疾眼快(lit. manos hábiles $\mathrm{y}$ ojos rápidos; fig. moverse o actuar con mucha rapidez), máo shǒu máo jiǎo 毛手毛脚 (lit. mano y pie ásperos; fig. descuido al sostener las cosas), zhǐ shǒu huà jiăo指手画脚(lit. señalar la mano y dibujar el pie; fig. criticar o dar órdenes de manera sumaria), gāo tái guì shǒu高抬贵手(lit. (uno) levantar su mano privilegiada; fig. (pedir a uno que) muestre su misericordia hacia otro), fān shǒu wéi yún fù shǒu wéi $y \check{u}$ 翻手为云覆手为雨(lit. producir nubes con un giro de la mano y la lluvia con otra; fig. (uno) actuar de manera contradictoria consigo mismo).

Las asociaciones semánticas de shǒu手 pueden resumirse con la figura de la página siguiente. 


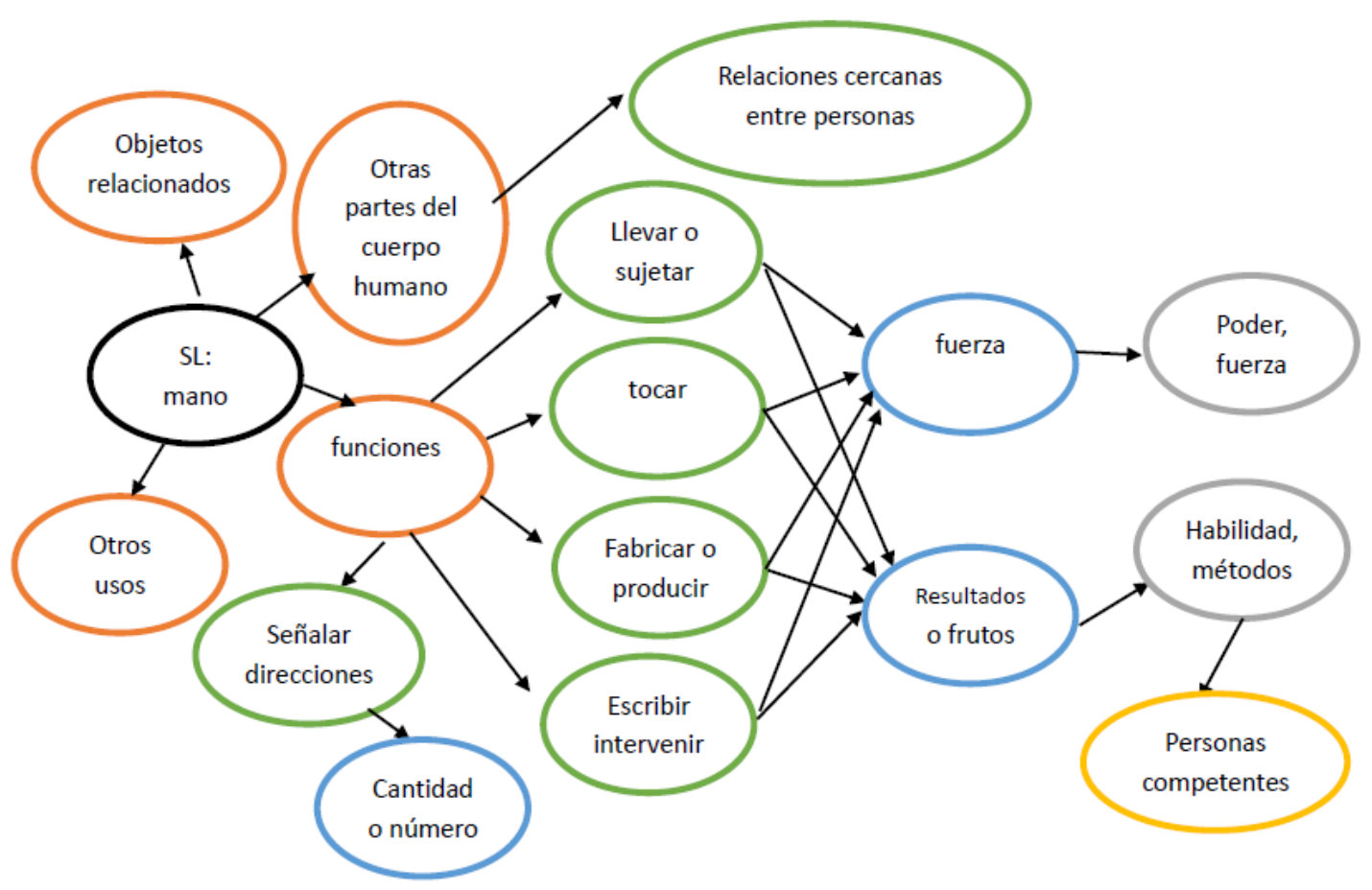

3. Estudio cognitivo de 'mano'

El término 'mano', en español presenta las siguientes asociaciones semánticas:

a. SL: mano como una parte del cuerpo humano;

b. SF: se asocia con los objetos de forma o función parecida a ella;

c. SF: se asocia con la función de señalar direcciones;

d. SF: se asocia con las acciones realizadas a través de ella: tocar y la sensación táctica, ayudas, protección, intervenir, pedir en matrimonio, criticar a alguien, etc.;

e. SF: se asocia con las fuerzas y el poder;

f. SF: se asocia con la habilidad, capacidad y los métodos;

g. SF: se asocia con las personas que poseen alguna habilidad;

h. SF: se asocia con el clasificador para jugar a las cartas o al ajedrez;

i. SF: se asocia con los términos relacionados con el fútbol;

j. SF: se asocia con los términos relacionados con la industria textil; 
k. SF: se asocia con otros clasificadores.

\begin{tabular}{|c|c|}
\hline Acepciones & Ejemplos \\
\hline $\begin{array}{l}\text { a. SL: mano como una parte del cuerpo } \\
\text { humano }\end{array}$ & ¡Manos arriba! \\
\hline $\begin{array}{l}\text { b. SF: se asocia con los objetos de forma o } \\
\text { función parecida a ella }\end{array}$ & Mano de la puerta \\
\hline $\begin{array}{l}\text { c. SF: se asocia con la función de señalar } \\
\text { direcciones }\end{array}$ & $\begin{array}{l}\text { Mano de reloj } \\
\text { A mano izquierda/derecha } \\
\text { Mano de las puertas } \\
\text { A la mano }\end{array}$ \\
\hline $\begin{array}{l}\text { d. SF: se asocia con las acciones } \\
\text { realizadas a través de ella }\end{array}$ & \\
\hline Tocar y sujetar & A mano armada \\
\hline Ayudar & Echar una mano a alguien \\
\hline Proteger & Dar la mano a uno \\
\hline Intervenir & Meter la mano en un asunto \\
\hline Pedir en matrimonio & Pedir la mano a alguien \\
\hline Criticar & Dar una mano a alguien \\
\hline \multicolumn{2}{|l|}{ e. SF: se asocia con las fuerzas y el poder } \\
\hline Fuerzas & Mano de obra, escasez de mano \\
\hline Poder e influencia & Mano blanda/dura/de hierro, tener mano con uno \\
\hline $\begin{array}{l}\text { f. SF: se asocia con la habilidad, } \\
\text { capacidad y los métodos }\end{array}$ & No tengo manos para tanto trabajo. \\
\hline $\begin{array}{l}\text { g. SF: se asocia con las personas que } \\
\text { poseen alguna habilidad }\end{array}$ & $\begin{array}{l}\text { Los retratos por la misma mano. } \\
\text { Toda la herencia fue a parar a sus manos. }\end{array}$ \\
\hline $\begin{array}{l}\text { h. SF: se asocia con el clasificador para } \\
\text { jugar a las cartas o ajedrez }\end{array}$ & $\begin{array}{l}\text { Echar una mano a las cartas } \\
\text { Tiene una buena mano esta vez. } \\
\text { ¿Quién es mano? }\end{array}$ \\
\hline $\begin{array}{l}\text { i. SF: se asocia con los términos } \\
\text { relacionados con el fútbol }\end{array}$ & Esta vez, me toca a mí ser mano. \\
\hline j. SF: se asocia con otros clasificadores & $\begin{array}{l}\text { Tienes que dar varias manos a este suelo tan sucio. } \\
\text { Una mano de vino. } \\
\text { Dar una mano de bofetadas/palos a alguien. }\end{array}$ \\
\hline
\end{tabular}

El término 'mano' en español es igual al chino, como una parte del cuerpo humano, aunque a veces también se refiere a la extremidad o los dos pies delanteros de algunos animales. Además, algunos instrumentos de contrucción también pueden ser 
denominados con esta palabra, como por ejemplo los asideros que se dejan en los paramentos de un sillar han sido llamados por el término 'mano'.

Sabemos que a través de las manos, podemos realizar muchas actividades, es decir, que las manos tienen muchas funciones, una de ellas la de indicar o señalar direcciones, por ejemplo: mano de reloj, a la mano izquierda/derecha, mano de las puertas, a la mano.

Otras funciones realizadas por esta parte corporal son las siguientes: tocar y sujetar: a manos armadas; ayudar: echar una mano a alguien; proteger o tener piedad hacia alguien: dar la mano a uno; intervenir un asunto: meter la mano en un asunto; pedir en matrimonio: pedir la mano a alguien; criticar a alguien: dar una mano a uno, etc.

Durante el proceso de realizar acciones con las manos, obviamente se aplica la fuerza, por eso, se puede asociar con la fuerza: mano de obra, escasez de manos, etc.; con el poder e influencia: mano dura/blanda/de hierro, tener mano con uno, tiene mucha mano en el ministerio, etc.

Al mismo tiempo, usar las manos para realizar las acciones implica poseer cierta fuerza y aplicar algún método, así que se puede asociar con la hablidad, la capacidad y los métodos: No tengo manos para tanto trabajo.

Además, las personas que poseen dichas fuerzas y habilidades pueden ser consideradas como competentes o que tienen alguna destreza, por lo tanto, se puede asociar con las personas competentes o cualificadas en algún campo: los retratos por la misma mano, toda la herencia fue a parar a sus manos, etc.

Muchos términos sobre los juegos realizados con las manos también usan esta palabra para algunas expresiones, como, por ejemplo, en los juegos de cartas y ajedrez existen expresiones como: echar una mano de ajedrez, tiene una buena mano esta vez, ¿Quién es mano? etc.; en el fútbol: esta vez, me toca a mí ser mano.

Como las manos pueden realizar la acción de contar el número y la cantidad, se las puede asociar con el uso como clasificador para contar los objetos, los números, las capas y las veces: tienes que dar varias manos a este suelo tan sucio, una mano de vino, dar dos manos de pintura, dar una mano de bofetadas/palos a alguien, etc.

En la figura de la página siguiente, resumimos las asociaciones semánticas de mano. 


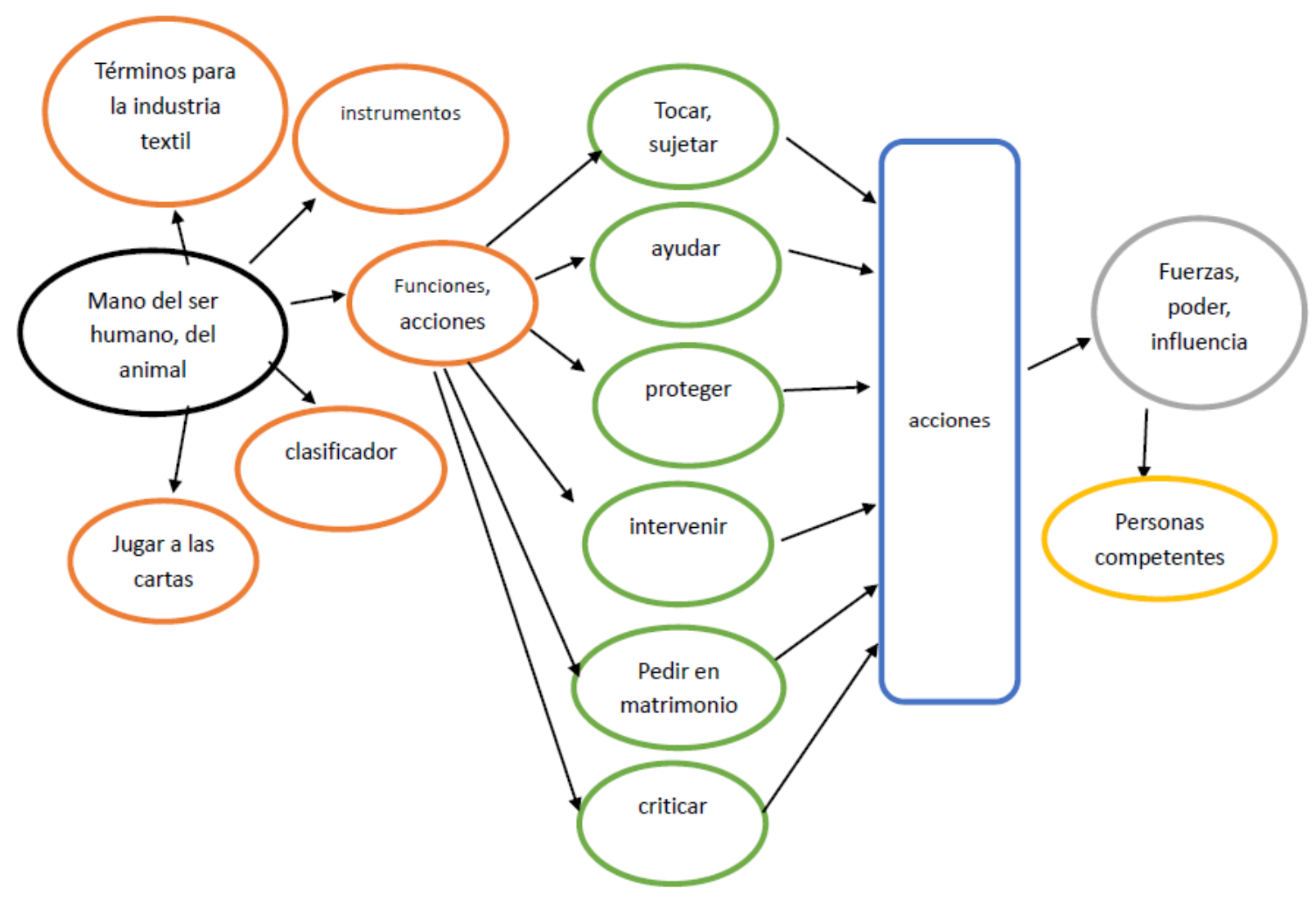

4. Comparación cognitiva de shǒu手 mano en chino y en español

En el estudio-aprendizaje de lenguas extranjeras, la comparación de los dos idiomas es un método muy eficiente e importante. Porque siempre y cuando conozcamos las similitudes y las diferencias entre las dos lenguas, podremos conseguir las estrategias de aprendizaje e investigación según las características propias de cada una de ellas. Tomando el caso de shǒu手mano, podemos ver lo semejante y lo diferente de este término en las dos lenguas.

\subsection{Comparación de la categoría gramatical}

Tanto en chino como en español, el término shǒu f fmano tiene la categoría gramatical de sustantivo, y debe mencionarse que esta voz en chino no tiene género, mientras que en español es un sustantivo femenino. 
En chino, hay expresiones de esta palabra sobre el uso de clasificador para contar la capacidad y los cambios de derechos y de posesiones, mientras que en español existen usos como clasificadores, pero es un sustantivo femenino.

Pueden verse en la siguiente tabla las comparaciones, en cuanto a categoría gramatical, de este término en los dos idiomas:

\begin{tabular}{|l|l|l|}
\hline & sustantivo & clasificador \\
\hline chino & + & + \\
\hline español & + femenino & - \\
\hline
\end{tabular}

(tabla 1. + uso existente; - uso no existente)

\subsection{Comparación de las asociaciones semánticas}

\subsubsection{Usos comunes de las asociaciones semánticas en chino y en español}

En primer lugar, en los dos idiomas el sentido literal signifca 'la mano del ser humano'.

En ambas lenguas, este término tiene la asociación de señalar o indicar la dirección, como en las mismas expresiones: 左/右手边a mano izquierda/derecha, 手头上a la mano, etc.

Tanto en chino como en español, hay asociaciones con las fuerzas, el poder y las influencias. Por ejemplo, en chino se dice $y \bar{\imath}$ shǒu zhe tiān一手遮天(lit. una mano que cubre el cielo; fig. ocultar la verdad al público o engañar al público mediante el poder), shǒu wú fù jī zhī lì 手无缚鸡之力(lit. las manos son tan débiles que no tienen fuerza para agarrar la gallina; fig. ser muy débil físicamente), shǒu wú cùn tiě手无寸铁(lit. no tener ningún arma en la mano; fig. estar totalmente desarmado), etc.; mientras tanto, en español se dice: mano dura/blanda/de hierro, tener mano con uno, tiene mucha mano en el ministerio, etc.

Al mismo tiempo, cuentan con las asociaciones sobre habilidades y capacidades, como por ejemplo en chino se dice miào shǒu hui chūn妙手回春(lit. con la mano hábil volver la primavera; fig. (un médico experto) devolver la vida de un paciente que está al borde de la muerte), xīn líng shǒu qiăo心灵手巧(lit. alma sensible y mano hábil; fig. (uno) 
inteligente y hábil) y en español: no tener tantas manos para algo, tener mano para el arte.

Además, en estos dos idiomas, esta palabra este término tiene asociaciones con las personas competentes o que tienen algunas habilidades, más concretamente en chino hay expresiones como: con las personas que tienen estas habilidades en sentido positivo (profesión): gē shǒu歌手(lit. mano para cantar; fig. cantante), sài chē shǒu赛车手(lit. mano para carreras de automóviles; fig. piloto de Fórmula 1), etc.; neutral: xuăn shǒu选手(lit. mano para elegir; fig. candidato), bāng shǒu帮手(lit. mano para ayudar; fig. ayudante), etc.; y negativo: pá shǒu扒手(lit. mano para escarbar; fig. ladrón o ratero), guì zi shǒu会//子手(lit. mano para cortar; fig. verdugo), etc.; mientras que en el español existen expresiones como 'los retratos por la misma mano', aunque no tienen usos tan amplios como el caso chino.

Cabe mencionar que, por las funciones y acciones realizadas por las manos tanto en chino como en español, lo común entre ambos idiomas son los siguientes: tocar y sujetar, producir y fabricar, ayudar, etc. Pueden consultarse los ejemplos expuestos anteriormente en el texto.

Por útlimo, en el caso de jugar a las cartas, en ambos idiomas existe la expresión de一手好牌una mano de cartas, aunque en el caso chino, se usa como un clasificador.

En fin, los puntos en común de las asociaciones semánticas de este término en chino y en español pueden ser los siguientes:

a. SL: mano del ser humano;

b. SF: se asocia con direcciones:

c. SF: se asocia con fuerzas, poder e influencias;

d. SF: se asocia con habilidades y capacidades;

e. SF: se asocia con las personas competentes o que dominan estas habilidades;

f. SF: se asocia con las acciones realizadas con manos: tocar y sujetar, producir y fabricar, ayudar, etc.;

g. SF: se asocia con el juego a las cartas. 
Pueden ser resumidos los usos comunes sobre las asociaciones semánticas en los dos idiomas con la figura de la página siguiente.

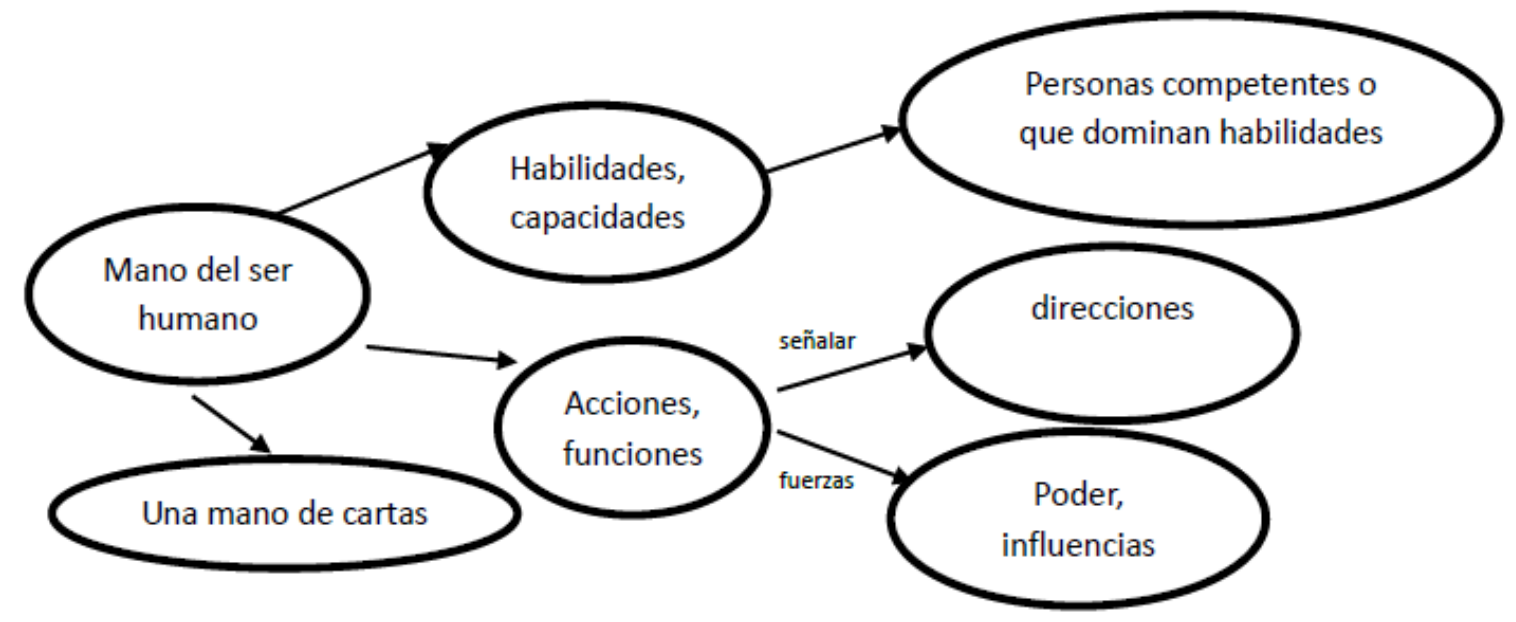

\subsubsection{Diferencias entre las asociaciones semánticas en chino y en español}

En español, la palabra 'mano' no solo significa la mano del ser humano, también puede usarse para expresar las extremidades o los pies delanteros de algunos animales, incluso puede ser la nariz del elefante. Mientras tanto, en el caso chino no existen tales acepciones.

A partir de los sentidos figurados, el término chino puede ser asociado con otras partes del cuerpo humano, como por ejemplo: shǒu zh亡̌手指( dedo), shǒu wàn 手腕(muñeca), shǒu zhăng手掌(palma), etc., pero en español no se encuentran estos usos o estas acepciones. También, esta palabra en chino tiene múltiples asociaciones relacionadas con los objetos vinculados con esta parte corporal: shǒujī 手机(lit. máquina de mano; fig. móvil); shǒu biăo 手表(lit. reloj de mano; fig. reloj de muñeca o de pulsera); shǒu zhuó 手镯(lit. pulsera de mano; fig. pulsera), etc.; en español son más escasas.

Además, la mano está compuesta de carne, sangre y hueso, lo cual simboliza vínculos estrechos. Por eso, en chino existen asociaciones sobre las relaciones cercanas como las que hay entre familiares y amigos, similares a uña y carne en el español: qíng tóng shǒu zú 情同手足(lit. tener el mismo sentimiento que el que hay entre las manos y los pies; fig. tener una relación tan cercana entre los amigos como la que se tiene con los familiares), xiōng dì rú shǒu zú 兄弟如手足(lit. los hermanos son como las manos y los 
pies; fig. tener una amistad tan cercana como el amor fraternal), shǒu xīn shǒu bèi dōu shì ròu手心手背都是肉(lit. tanto la palma como el dorsal de la mano son carne de uno; fig. no poder elegir cuál es su favorita entre dos personas a las que se quiere), etc., pero en el caso español no existen este tipo de asociaciones.

Sin embargo, en el caso de español, existen asociaciones sobre los juegos deportivos, como el fútbol, las cartas y el ajedrez, y además, se puede asociar con los términos relacionados con la industria textil. También, en el caso de los términos de los juegos como cartas y ajedrez, el español tiene más usos que el chino, pueden resumirse las diferencias de las asociaciones semánticas sobre este término en los dos idiomas con la siguiente tabla:

\begin{tabular}{|c|c|c|}
\hline & chino & español \\
\hline SL: extremidades o pies delanteros de los animales & - & + \\
\hline SF: otras partes del cuerpo humano relacionadas con la mano & + & - \\
\hline SF: objetos relacionados con la mano & + & - \\
\hline SF: relaciones cercanas como las que hay entre familiares y amigos & + & - \\
\hline SF: términos sobre el fútbol y la industria textil & - & + \\
\hline SF: términos sobre juegos como las cartas y el ajedrez & - & + \\
\hline
\end{tabular}

(tabla 2. + uso existente; - uso no existente)

\section{Conclusión}

Luque Durán (2001) nos explica en su obra que en el léxico de los lenguajes mundiales existen aspectos universales y particulares. En nuestro caso de polisemia en chino y en español, más concretamente en el caso del término 'mano (shǒu f)', se puede decir que hay tendencias generales, mientras que no se descartan las diferencias entre los dos idiomas. A través de este estudio, ofrecemos una posiblidad, en investigaciones futuras, de declarar las diferentes clasificaciones del léxico en ambas lenguas, como por ejemplo la polisemia en las palabras del cuerpo humano, de los animales, de los colores, de los adjetivos de dimensiones, etc. 
Bibliografía

Cuenca, María Josep y Hilferty, Joseph (2013): Introducción a la lingüítica cognitiva. Barcelona: Ariel Letras, pág. 125-149.

Luque Durán, Juan de Dios (2001): Aspectos universales y particulares del léxico de las lenguas del mundo. Granada: Granada Lingvistica.

Pottier Navarro, Huguette (1991): La polisemia léxica en español. Madrid: editorial Gredos, pág.14-25.

Zhāng, Shào Quán 张绍全 (2010): 学习者多义词习得的认知语言学研究 (Chinese English Learners' Acquisition of English Polysemous Words: A Cognitive Linguistic Approach). Chongqing: Chongqing University Press.

Recibido: 1 de diciembre de 2015

Aceptado: 19 de mayo de 2016

Revisado: 28 de mayo de 2016

Publicado: 301 de mayo de 2016 